\title{
2.5D Infrared Range and Bearing System for Collective Robotics
}

\author{
James F. Roberts, Timothy S. Stirling, Jean-Christophe Zufferey and Dario Floreano \\ (Pre-print version, Published at IROS2009)
}

\begin{abstract}
In the growing field of collective robotics, spatial co-ordination between robots is often critical and usually achieved via local relative positioning sensors. We believe that range and bearing sensing, based on infrared technology, has the potential to fulfil the strict requirements of real-world collective robots. These requirements include: small size, light weight, large range, high refresh rate, immunity against tilting and misalignment, immunity against ambient light changes, and good range and bearing accuracy. Currently, there are no range and bearing systems that have been designed to cope with such strict requirements. This paper presents a custom range and bearing system, based on a novel cascaded filtering technology, complemented by hybrid infrared/Radio Frequency (RF) communication, which has been designed specifically to meet all these expectations. The system has been characterised and tested, proving its viability.
\end{abstract}

\section{INTRODUCTION}

Relative positioning [1] is an important sensing system allowing spatial coordination in collective and swarm robotics [2]-[4]. However, no commercial sensor exists that provides relative positioning at a reasonable size and weight. Some researchers have developed custom solutions, but they all present strong limitations. This paper describes a system that alleviates some of these limitations. In particular, we have been looking at the misalignment that may arise when using such sensors on flying platforms that can tilt or be at slightly varied altitudes. The same problem may arise with terrestrial robots when moving in rough terrain.

For effective deployment of a robot swarm, especially a swarm of Miniature Aerial Vehicles (MAVs) [5], the relative positioning system must be light weight, small and low powered. A system with a high refresh rate is preferable in order to allow greater scalability towards large swarms due to faster signal acquisition times, and to give accurate results for fast moving robots like MAVs. Additionally, a long range sensor solution is advantageous as it increases the coverage area of a robot swarm, e.g. in surveillance or search tasks, by increasing the potential separation distance between robots. Terrestrial robots operating in environments with uneven floors may experience pronounced tilting of the sensory plane or operate at slightly different heights, which we refer to as misalignment $(2.5 \mathrm{D}$ is defined as $2 \mathrm{D}$ sensing with tilting and misalignment immunity). Moreover, a hovering MAV (e.g. [5]) during a horizontal translation will typically tilt 10 to 20 degrees in the direction of travel in order to produce the required lateral forces. This tilting or misalignment can

Laboratory of Intelligent Systems (LIS), Ecole Polytechnique Fédérale de Lausanne (http://lis.epfl.ch), Lausanne, 1015, Switzerland. james.roberts@epfl.ch

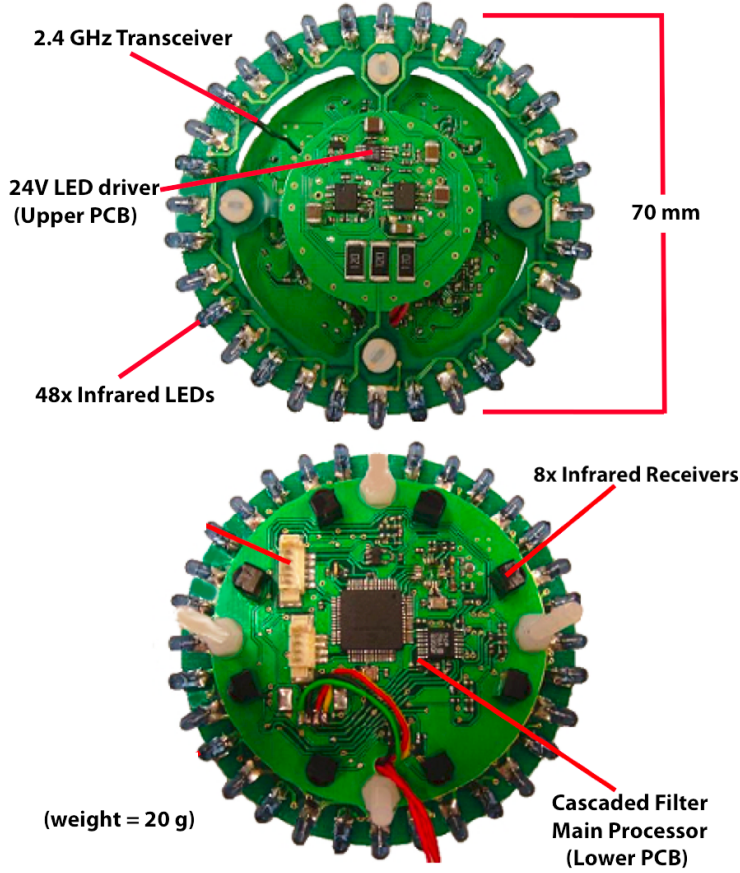

Fig. 1. Left: top view of the Range and Bearing Infrared Transceiver (RABIT) sensor, right: bottom view of RABIT sensor

give erroneous relative position estimations or even total loss of signal. Robots in the real-world will also encounter large changes in ambient light when moving from indoors to outdoors and between rooms. These are features that have not been addressed by existing $2 \mathrm{D}$ relative positioning systems [1]-[4].

For applications on swarms of small robots and MAVs, there are important sensor specification requirements, we define a practical set of sensor specifications as follows: maximum sensor diameter of $8 \mathrm{~cm}$ and weight of $30 \mathrm{~g}$, range of up to $5 \mathrm{~m}$ allowing for good robot displacement, a refresh rate of better than $10 \mathrm{~Hz}$ for a swarm of 50 robots, immunity against misalignment and tilting up to $\pm 20^{\circ}$ (2.5D), immunity against ambient light changes up to 10,000 lux, accuracy better than $15 \%$ for both range and bearing.

Various relative positioning technologies are available that use different sensing modalities, however, to date there are no sensor solutions accommodating all the specifications as stated above. For example, ultrasound can be used to achieve high accuracy range and bearing measurements [6], [7], but with a relatively slow refresh rate $\left(\frac{1}{0.075 . N} \mathrm{~Hz}\right.$ for $N$ robots, see [7]), due to slow sound dissipation. Therefore, 
it is not suitable when large numbers of robots need to be synchronised to avoid interference and sensor crosstalk. The Received Signal Strength Indicator (RSSI) of wireless ethernet is used in [8], but this requires detailed a-priori knowledge of the operating environment to reduce the noisy effects of environmental interference. Many other systems exist that depend on complex processing with cameras [9], or other heavy hardware [10].

In contrast, using infrared signals as a medium for relative positioning has the benefits of high update rates $(1 \mathrm{kHz})$ and small low cost hardware. The performance of such sensors has been validated in several multi-robotic systems [2], [3]. The maximum range of these sensors is usually up to 2.5 m [3] or $3.0 \mathrm{~m}$ [1], with refresh rates up to $25 \mathrm{~Hz}$ for 10 robots, where the refresh rate is inversely proportional to the number of robots to allow for signal dissipation.

Considering the benefits of high update rates and small light hardware, an infrared based relative positioning system is appropriate to enable swarming of small robots. To the best of our knowledge, the only on-board relative positioning system for an indoor flying robot is presented by Melhuish and Welsby [11] for use on a swarm of lighter than air vehicles (LTAV). In their work, an infrared relative positioning system with $5 \mathrm{~m}$ range, based on the design by Kelly and Keating [12], is used on a flying robot swarm to achieve a simple gradient ascent behaviour towards an emitting beacon. None of the papers mentioned above provide technical or characterisation details such as accuracy, noise, communication bandwidth, or ability to handle robot tilting. Moreover, their system is frequency division multiplexed providing a unique carrier frequency for each robot. This method is not scalable to large swarms, as there are a limited number of communication channels available within the defined frequency spectrum.

We believe that range and bearing sensing, based on infrared technology, has the potential to fulfil the strict requirements of real-world collective robots. This paper presents a custom range and bearing system, called Range and Bearing Infrared Transceiver (RABIT) (fig. 1), based on a novel cascaded filtering technology, complemented by hybrid infrared/Radio Frequency (RF) communication. We describe our approach for increasing the reliable working range using cascaded amplification and filtering, improving robustness to robot tilting by the use of multiple LED rings and increasing scalability for larger robot swarms by increasing the sensor refresh rate. We first describe the sensor technology with emphasis on the specific requirements for robotic platforms that may experience relative tilting, misalignment and large changes in ambient light. We then present characterisation and testing experiments.

\section{SENSOR DESIGN}

Relative positioning systems based on infrared technology, typically use RSSI measurements from multiple infrared receivers to calculate the relative range and bearing of a transmitting robot. The receiver with the strongest RSSI measurement and the left and right adjacent receivers are used to

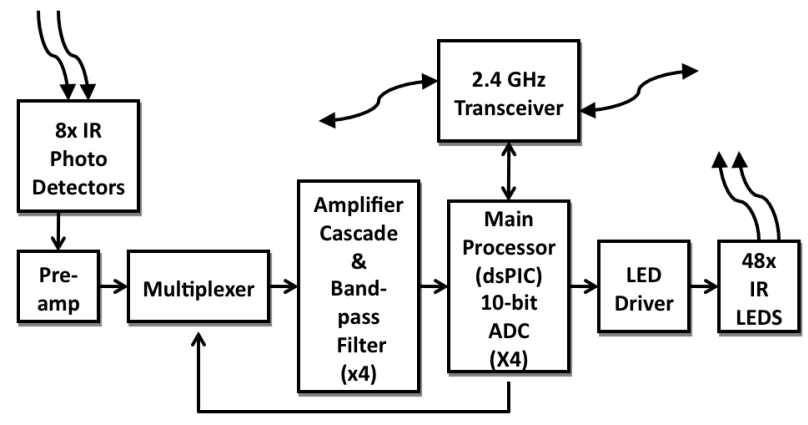

Fig. 2. System diagram of a single RABIT sensor showing infrared signal path and coupled RF transceiver for speeding up RSSI measurements

triangulate the bearing angle. The spacing of the transmitters and receivers, and their beam width, is very important to obtain a good $360^{\circ}$ coverage. It is important to obtain a RSSI measurement with a good signal to noise ratio and a large dynamic range, which determines the sensors maximum range and resolution. A standard method in measuring RSSI is to use a RF chip equipped with a RSSI pin. These chips are designed for radios, so they operate in the $\mathrm{MHz}$ band. This is a problem as it requires complex circuitry and the RF interference can be difficult to work with on small Printed Circuit Boards (PCB). These chips tend to have a relatively small dynamic range that limits the performance. Also, the data is Frequency Modulated (FM) and sent over the audio channel which limits the sensors maximum speed. A turn taking algorithm must be employed to separate individual infrared transmissions to prevent crosstalk and erroneous readings. Therefore, the speed of the system is dependant on the time it takes to reliably measure the RSSI and receive the transmitted data, the later taking the majority of the time.

Our RABIT sensor (fig. 1) uses a combination of new techniques to optimise the way a RSSI measurement is attained and how it transmits the data. To obtain a RSSI with an increased dynamic range we use a four stage cascaded amplifier. Each of the four stages is designed to output a voltage corresponding to a complementary region of the maximum range. A block diagram of the system can be seen in fig. 2. To optimise the speed of a RSSI measurement we remove the data from the infrared signal and transmit it over a $2.4 \mathrm{GHz}$ transceiver. The transceiver is used to synchronise each RABIT sensor by implementing a simple turn taking algorithm. The details of the infrared transmission, infrared reception, cascaded signal amplification, range and bearing calculation and communication are outlined below.

\section{A. Infrared Transmission}

The transmitter sends a burst of $950 \mathrm{~nm}$ wavelength infrared pulses at a carrier frequency of $455 \mathrm{kHz}$. This has been chosen as it is the high-speed standard for infrared modules and it is easy to obtain small and cheap ceramic filters at this frequency. The maximum range of the system is determined by both the transmission power and the receiver sensitivity. Therefore, to optimise maximum range it is important to use high powered infrared LEDs. Additionally, the beam width 


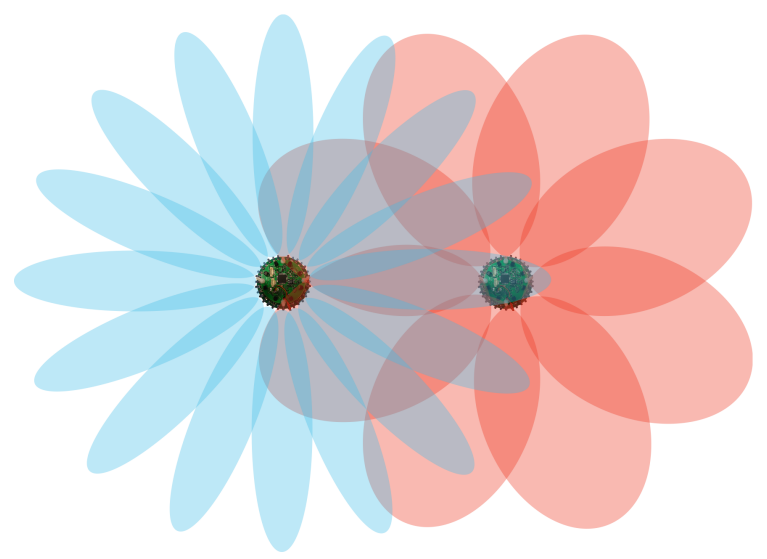

Fig. 3. Plot representing an approximate half angle intensity of the 16 narrow beam $\left( \pm 25^{\circ}\right)$ transmitters (left), and the approximate half angle viewing of the 8 wide beam $\left( \pm 60^{\circ}\right)$ receivers (right)

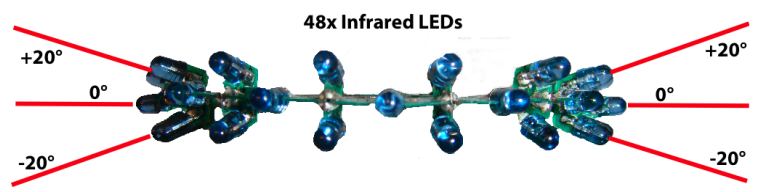

Fig. 4. Side view of the RABIT sensor showing the placement of the three $360^{\circ}$ infrared LED rings, giving $\pm 20^{\circ}$ vertical coverage, each line represents the centre of the LEDs radiated $\pm 25^{\circ}$ beam

of the infrared LED is important. If the LED has a smaller beam width, then the projected infrared light will be more focused and thus have a further range. There is a compromise between maximum range and the number of LEDs required for full $360^{\circ}$ coverage. The more focussed the beam, the higher the number of required LEDs. To obtain good $360^{\circ}$ coverage we decided to use a ring of 16 TSAL4400 LEDs from Vishay which have a half angle intensity of $\pm 25^{\circ}$ (fig. $3)$, giving a small overlap $\left(2.5^{\circ}\right)$.

For a robust system, it is necessary to have a mechanism to cope with tilting or slight misalignment between RABIT sensors. As the sensor is tilted, the signal strength changes corresponding to both the transmitters half angle intensity and receiver half viewing angle. As the infrared LEDs are optimised for extended range, they have a small beam width $\left( \pm 25^{\circ}\right)$ that causes the power intensity to reduce rapidly, even with small tilt angles. To overcome this problem, our system has two additional LED rings tilted up an down to increase the vertical coverage (fig. 4).

\section{B. Infrared Reception}

There are eight photodetectors (BPV22NF from Vishay) evenly spaced about the same axis as the infrared LEDs (fig. 3). The photodetectors convert the infrared pulses into a small electrical current. This small signal is then preamplified and fed into a multiplexer that handles the eight receiver signals. It is important to obtain the best possible signal from the sensor, as the signal to noise ratio is propagated through the rest of the receiver system. Therefore, noise filters have been placed on all analog power supply pins and component placement has been done very carefully.

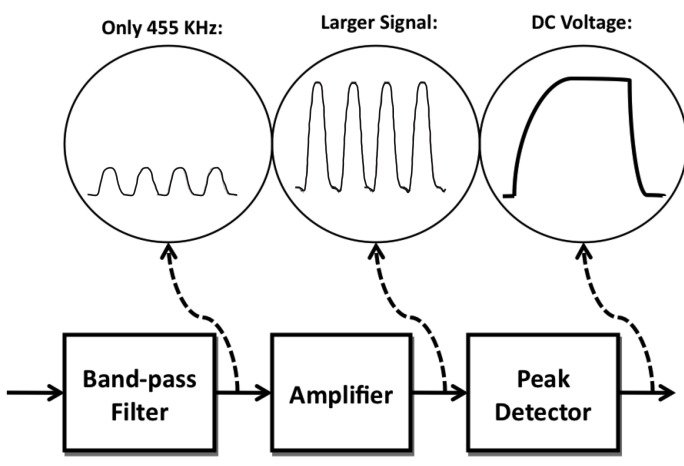

Fig. 5. Block diagram of a single filter cascade stage showing the outputs of the bandpass filter, amplifier and peak detector

Choosing a high sensitivity photodetector and a low-noise, high-speed pre-amplifier is also important.

Existing systems can have problems with ambient light and may not work well in bright rooms or outdoors. This is due to the high gain of the pre-amplifier causing saturation as the bias voltage on the photodetector diode increases. To minimise this effect we have placed AC coupling directly on the photodetector output before the pre-amplifier. This means that saturation will occur only if the light intensity goes beyond the limits of the reverse-biased photodetector.

\section{Cascaded Signal Amplification}

The RABIT uses a cascaded amplification system (fig. 5) to obtain the RSSI measurement. This method is an important difference between our solution and existing ones. The pre-amplified signal from the photodetector is fed into a series of cascaded amplifiers. The gain of each amplifier is precisely tuned to cover a specific region of the maximum range. By splitting up the range into smaller regions we effectively increase the range resolution of the sensor and in-turn also increase the bearing resolution. On each stage of the cascade there is a bandpass filter tuned to detect the $455 \mathrm{kHz}$ carrier frequency of the transmitter. This ensures we only receive the desired transmission signal and reject noise and interference from within the circuit, sensors and most importantly external infrared devices. Care must be taken when tuning the gains to prevent the last stage of the cascade from ringing. Ringing occurs when the cascade starts to resonate at the filter frequency due to the high amplification of the noise floor. A half-bridge peak signal detector then converts the positive side of each cascade carrier frequency output to a DC voltage. The output voltage of each cascade is sampled by the internal 10-bit Analog to Digital Converter (ADC) of the main processor (dsPIC). The typical output of the RABIT sensors tuned amplifier cascade can be seen in fig. 6 .

\section{Calculating Range \& Bearing}

Before calculating the range and bearing it is necessary to create a look-up table to combine the four cascade outputs and perform the range interpolation. First, the gains of the cascades have to be selected so that there is a small overlap 


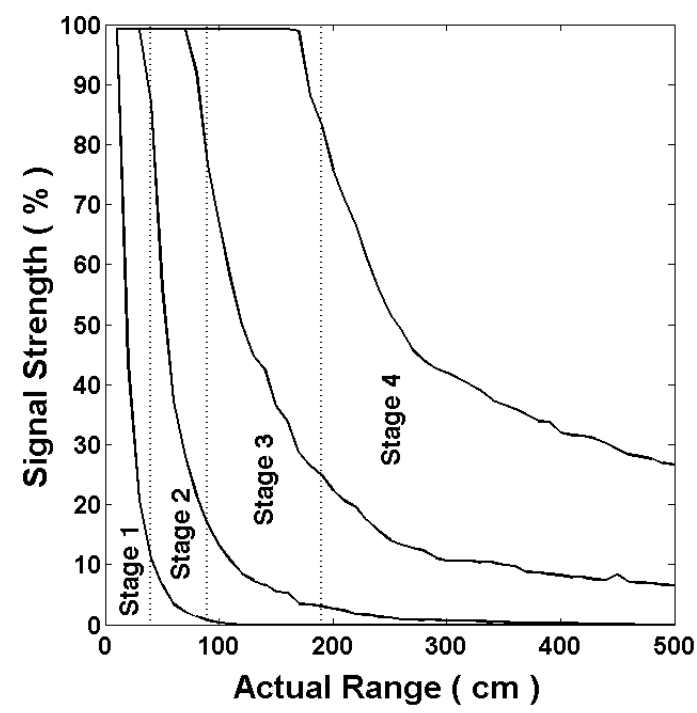

Fig. 6. Typical output response of the four cascaded filtering stages after characterisation and gain tuning. Note: the highest slope section of each stage covers a defined region of the range and that there is a small overlap between stages to ensure the robustness of the system

in the range segmentation (fig. 6). This is done in hardware by selecting the appropriate resistor for the required gain. Second, the look-up table is created by taking samples over the full sensor range from $0 \mathrm{~cm}$ up to $500 \mathrm{~cm}$. The appropriate cascade number and ADC value is recorded at $10 \mathrm{~cm}$ intervals. 100 samples are averaged to obtain a single point of measurement. These ADC values correspond to the RSSI for each of the eight receivers. The look-up table is then used to interpolate the linear distance information from the non-linear measured RSSI values. The four RSSI measurements from each cascade are combined using the look-up table to obtain a single relative range. In order to calculate the bearing information a triangulation method is used [1]. This is done by searching each of the eight range measurements to find the minimum range $r^{M}$ (i.e. largest RSSI). The immediate distances on the left and right sides of the $r^{M}$ are defined as $r^{L}$ and $r^{R}$, respectively. These three distances are used to triangulate the bearing angle. This bearing angle is then used to correct the original range estimation as the range is dependent on the angle. This gives both the relative range and the bearing information of the transmitting robot. The following equations define the range $(r)$ and bearing $(\theta)$ of each transmitting robot. We use the same algorithm as developed by Pugh and Martinoli [1]:

$$
r=\frac{1}{\sqrt{a^{2}+b^{2}}}, \quad \theta=\arctan \frac{b}{a}+Q
$$

where $Q$ is $2 \pi$ if the result of $\theta$ is negative, else zero. $a$ and $b$ are defined as:

$$
a=\frac{r^{L}+r^{R}+2 . r^{M}}{2 \cdot \cos \left(\frac{\pi}{4}\right)+2}, \quad b=\frac{r^{L}-r^{R}}{2 \cdot \sin \left(\frac{\pi}{4}\right)}
$$

Equation 2 expects each range term to be an RSSI measurement. Therefore, we can solve equation 2 by simply inverting the range measurements obtained by the look-up table, as they are inversely proportional to the RSSI.

\section{E. Communication}

The refresh rate of the system is directly dependent on the time it takes to reliably measure the RSSI of an infrared transmission. As far as we know, in existing relative positioning systems [1], [3], [11], [12], infrared is used to both find the RSSI and to transmit the communication data. However, transmitting infrared data over the range required is reasonably slow. When using standard $455 \mathrm{kHz}$ infrared modules designed for data transmission over the distances we require (up to $5 \mathrm{~m}$ ), the maximum reliable bit rate is only $20 \mathrm{Kbps}^{1}$. Unfortunately, the RSSI radio chips also have a speed limitation, as the data is sent over the audio channel; the maximum attainable speed is still only $\approx 20 \mathrm{Kbps}$.

Our new high-speed technique removes the data transmission from the infrared and sends data communication over a $2.4 \mathrm{GHz}$ chip, capable of speeds of up to $2 \mathrm{Mbps}$, which is significantly faster than using infrared. The data must be synchronised with the infrared transmissions for the system to function correctly. The infrared transmission is only used for RSSI measurements, thus allowing for the fastest possible refresh rate at the given infrared carrier frequency.

Our system employs a turn taking algorithm with adaptive time division multiplexing. When an infrared burst starts, the data transmission is triggered and is sent over the $2.4 \mathrm{GHz}$ RF link (fig. 2). Each RABIT will transmit on both mediums simultaneously in a given sequence defined by their unique ID number. When a wireless message is received, the infrared sampling is triggered and the timed multiplexer then samples each of the eight receivers after a peak in the infrared has been detected. After the last receiver is sampled, the procedure repeats for the RABIT with the next ID number. This is a simple and effective method for robust operation without implementing a more complex scaleable communication protocol such as Carrier Sense Multiple Access (CSMA) [1]. Currently tests have only been conducted using two RABIT sensors, however, the speed seems not to be an issue for us, as the estimated bandwidth of the sensor, based on the cascaded filter technique, is ample even when running 50 robots $(\approx 20 \mathrm{~Hz}$ update).

\section{SENSOR CHARACTERISATION}

A series of tests have been carried out to determine the accuracy of the range and bearing estimations and robustness to tilt and lighting changes. 50 measurements were taken at each position and averaged.

\section{A. Range Accuracy}

To test the RABIT sensor's range accuracy, an experiment using two RABIT sensors was conducted with one transmitting and one receiving. The position of the transmitting RABIT was fixed and the receiving RABIT was moved

\footnotetext{
${ }^{1}$ http://www.vishay.com/docs/82147/tsop7000.pdf
} 


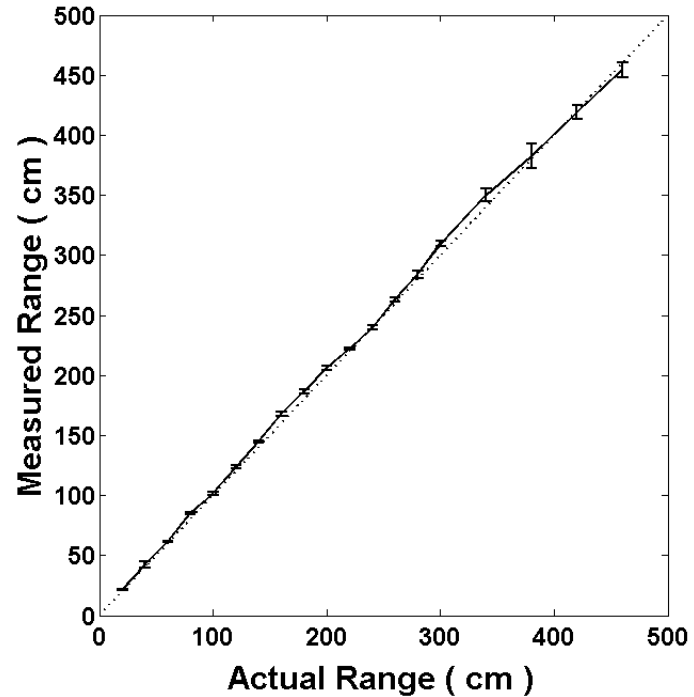

Fig. 7. Actual range vs. the mean of the measured ranges across all bearings: $0^{\circ}$ to $90^{\circ}$. The error bars represent the maximum standard deviation. 50 measurements were taken at each position

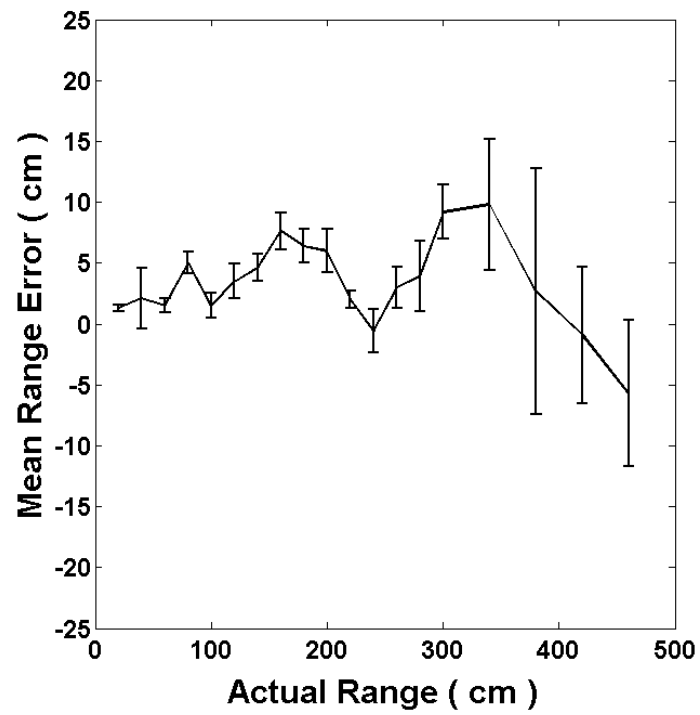

Fig. 8. Close up view of the range accuracy showing the mean of the measured ranges across all bearings: $0^{\circ}$ to $90^{\circ}$. The error bars represent the maximum standard deviation. 50 measurements were taken at each position

from $0 \mathrm{~cm}$ to $300 \mathrm{~cm}$ at $20 \mathrm{~cm}$ steps, and from $340 \mathrm{~cm}$ to $460 \mathrm{~cm}$ with $40 \mathrm{~cm}$ steps. To incorporate the effects of bearing changes on the range estimation we repeated the measurements for different bearings from $0^{\circ}$ up to $90^{\circ}$ at steps of $10^{\circ}$. The mean and the standard deviation of the measured ranges across all bearings can be observed in fig. 7. The plot shows the maximum standard deviation at each measured angle. The maximum mean distance deviation was $9.8 \mathrm{~cm}$ at $380 \mathrm{~cm}$, and the maximum distance error percentage was $6.5 \%$ at $20 \mathrm{~cm}$. A close up view of the range error is shown in fig. 8 .

Looking at fig. 8 we can see a change in the mean range error that is not smooth, however, the standard deviation is

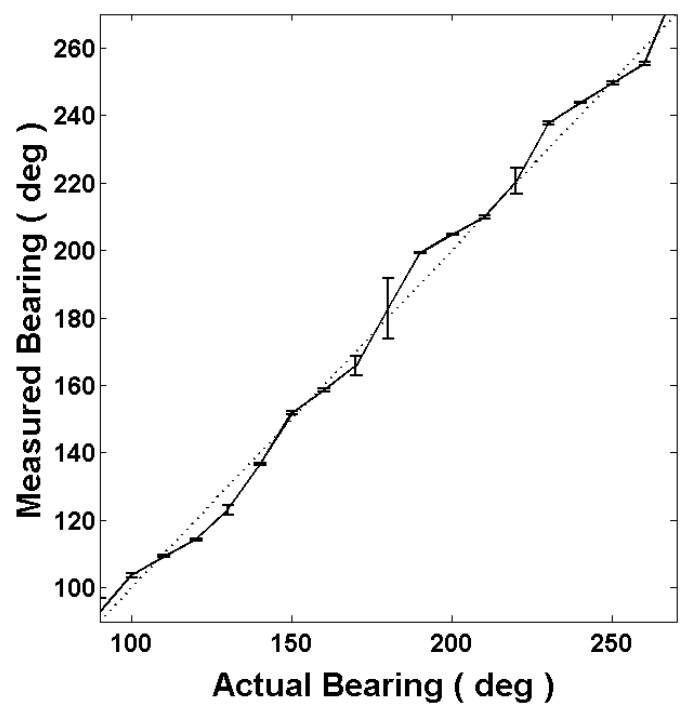

Fig. 9. Actual bearing vs. measured bearing (mean) for the ranges: $20 \mathrm{~cm}$, $100 \mathrm{~cm}, 300 \mathrm{~cm}$ and $400 \mathrm{~cm}$. The error bars represent the maximum standard deviation. 50 measurements were taken at each position

still very small. We believe that this change is mainly due to some small instabilities with ambient temperature change. We have observed that even with small changes in ambient temperature the cascade outputs start to shift. This means that the sensors output will no longer be correct as the look-up table calibration is shifted. This instability may be caused by the sensors main processor. It becomes hot to touch during operation and is located on the opposite side of the PCB where the sensitive cascaded filter is located.

\section{B. Bearing Accuracy}

To test the RABIT sensor's bearing accuracy the position of the transmitting RABIT was fixed and the receiving RABIT rotated between $90^{\circ}$ and $270^{\circ}$ at $10^{\circ}$ steps. We measured the bearing error at the following different ranges: $20 \mathrm{~cm}, 100 \mathrm{~cm}, 300 \mathrm{~cm}$ and $400 \mathrm{~cm}$. The mean and the standard deviation of the measured bearing across all ranges is shown in fig. 9. The plot shows the maximum standard deviation measured at each tested range. The maximum bearing deviation was $9.4^{\circ}$ giving a maximum bearing error (fig. 10) percentage of $2.6 \%$.

Looking at fig. 9 we can see there is a small bearing error that occurs in a sinusoidal pattern. We believe that this is due to the model of the photodetector not being accurate. The algorithm makes some assumptions about the photodetector response, this was expected and has been explained in [1].

\section{Tilt Immunity}

To test the RABIT sensor's immunity against tilting and misalignment, the position of the transmitting RABIT was fixed and the receiving RABIT placed at the following distances: $20 \mathrm{~cm}, 100 \mathrm{~cm}, 300 \mathrm{~cm}, 400 \mathrm{~cm}$ and $450 \mathrm{~cm}$. At each distance the tilt angle of the transmitting RABIT was adjusted to: $0^{\circ}, 10^{\circ}, 20^{\circ}, 30^{\circ}$. The percentage error change in recorded range at each distance, at the 3 tested tilt angles with respect to $0^{\circ}$, is shown in table I. At the targeted tilt 


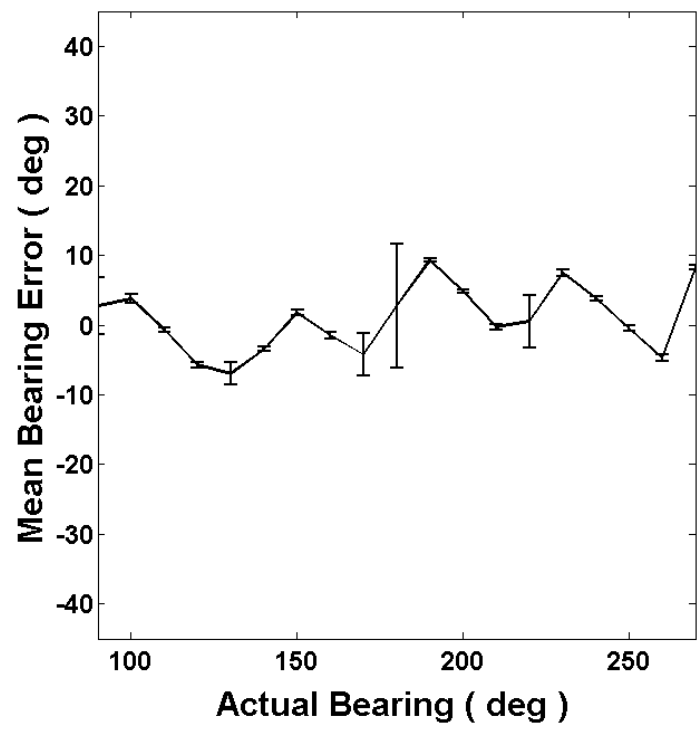

Fig. 10. Close up view of the bearing accuracy showing the mean of the measured bearings across the ranges: $20 \mathrm{~cm}, 100 \mathrm{~cm}, 300 \mathrm{~cm}$ and $400 \mathrm{~cm}$. The error bars represent the maximum standard deviation. 50 measurements were taken at each position

TABLE I

TILT IMMUNITY: PERCENTAGE ERROR CHANGE IN RECORDED RANGE AT $20 \mathrm{CM}, 100 \mathrm{CM}, 300 \mathrm{CM}, 400 \mathrm{CM}$ AND $450 \mathrm{CM}$, AT TILT ANGLES OF $10^{\circ}$, $20^{\circ}$ AND $30^{\circ}$ WITH RESPECT TO $0^{\circ}$

\begin{tabular}{|c|c|c|c|}
\hline \multirow{2}{*}{} & \multicolumn{3}{|c|}{ Tilt Angle: } \\
\cline { 2 - 4 } & $10^{\circ}$ & $20^{\circ}$ & $30^{\circ}$ \\
\hline $20 \mathrm{~cm}$ & $1.36 \%$ & $0.68 \%$ & $14.97 \%$ \\
\hline $100 \mathrm{~cm}$ & $3.69 \%$ & $6.38 \%$ & $16.89 \%$ \\
\hline $300 \mathrm{~cm}$ & $1.45 \%$ & $6.39 \%$ & $15.46 \%$ \\
\hline $400 \mathrm{~cm}$ & $3.61 \%$ & $3.33 \%$ & $8.64 \%$ \\
\hline $450 \mathrm{~cm}$ & $4.50 \%$ & $7.41 \%$ & $19.84 \%$ \\
\hline
\end{tabular}

angle of $20^{\circ}$ the maximum mean range error is $7.41 \%$ at $450 \mathrm{~cm}$.

\section{Light Immunity}

To test the system's immunity against large light changes, the position of the transmitting RABIT was fixed and the receiving RABIT placed at the following ranges: $20 \mathrm{~cm}$, $100 \mathrm{~cm}, 300 \mathrm{~cm}, 400 \mathrm{~cm}$ and $450 \mathrm{~cm}$. Measurements were taken at 0,610 , and 10,000 lux, which is equivalent to a dark room, office room and an overcast day outdoors, respectively. At all of these ranges we observed less than $1 \%$ error, therefore, we can state that the system is not affected by large ambient light changes due to $\mathrm{AC}$ coupling directly at the photodetector.

\section{CONCLUSIONS}

This paper presents a sensing solution that enables the possibility for practical swarm robot co-ordination. A custom range and bearing system based on a novel cascaded filtering technology, complemented by hybrid infrared/RF communication is proposed. The system measuring $70 \mathrm{~mm}$ in diameter and weighing $20 \mathrm{~g}$, has been fully characterised and tested with the following results: maximum range of up to $5 \mathrm{~m}$ allowing for good robot displacement, a theoretical refresh rate better than $15 \mathrm{~Hz}$ for a swarm of 50 robots, immunity against misalignment and tilting greater than $\pm 20^{\circ}$, immunity against ambient light changes up to at least 10,000 lux (indoor to outdoor), accuracy better than $7 \%$ and $3 \%$, for range and bearing respectively. Based on these results, all of the specifications have been fulfilled, suggesting that the system is a viable solution for collective robotics. The sensor can be further improved by fixing the temperature instability and using a more accurate model for the photodetector. In the future we plan to extend to a 3D system specifically for flying robots. Also, we would like to explore the possibility of using the same system for collision detection.

\section{ACKNOWLEDGEMENTS}

We would like to thank Jim Pugh and Alcherio Martinoli for their initial research support. This work is part of the Swarmanoid project, a Future Emerging Technologies (FET IST-022888) project funded by the European commission and the Swiss National Science Foundation.

\section{REFERENCES}

[1] J. Pugh, X. Raemy, C. Favre, R. Falconi, and A. Martinoli, "A Fast On-Board Relative Positioning Module for Multi-Robot Systems," IEEE/ASME Transactions on Mechatronics, Focused Section on Mechatronics in Multi Robot Systems, 2009.

[2] D. Payton, R. Estkowski, and M. Howard, "Pheromone robotics and the logic of virtual pheromones," in Swarm Robotics, vol. 3342. Springer Berlin / Heidelberg, 2005, pp. 45-57.

[3] J. S. J. McLurkin, "Distributed algorithms for dispersion in indoor environments using a swarm of autonomous mobile robots," in 7th International Symposium on Distributed Autonomous Robotic Systems, Toulouse, France, 2004.

[4] A. Howard, M. J. Mataric, and G. S. Sukhatme, "Mobile sensor network deployment using potential fields: A distributed, scalable solution to the area coverage problem," Distributed Autonomous Robotic Systems, vol. 5, pp. 299-308, 2002.

[5] J. F. Roberts, T. Stirling, J.-C. Zufferey, and D. Floreano, "Quadrotor using minimal sensing for autonomous indoor flight," in Proceedings of the 3rd US-European Competition and Workshop on Micro Air Vehicles, 7th European Micro Air Vehicle Conference and Flight Competition, 2007.

[6] S. Shoval and J. Borenstein, "Measuring the relative position and orientation between two mobile robot with binaural sonar," in ANS 9th International Topical Meeting on Robotics and Remote Systems, Seattle, Washington, March 2001.

[7] F. Rivard, J. Bisson, F. Michaud, and D. Letourneau, "Ultrasonic relative positioning for multi-robot systems," in Robotics and Automation, 2008. ICRA 2008. IEEE International Conference on, 2008, pp. 323 328.

[8] A. M. Ladd, K. E. Bekris, A. Rudys, L. E. Kavraki, and D. S. Wallach, "Robotics-based location sensing using wireless ethernet," Wireless Networks, vol. 11, no. 1-2, pp. 189-204, January 2005.

[9] T. Nakamura, M. Oohara, T. Ogasawara, and H. Ishiguro, "Fast selflocalization method for mobile robots using multiple omnidirectional vision sensors," Mach. Vision Appl., vol. 14, no. 2, pp. 129-138, 2003.

[10] L. Montesano, L. Montano, and W. Burgard, "Relative localization for pairs of robots based on unidentifiable moving features," in Intelligent Robots and Systems, 2004. (IROS 2004). Proceedings. 2004 IEEE/RSJ International Conference on, vol. 2, 2004, pp. 1537-1543.

[11] C. Melhuish and J. Welsby, "Gradient ascent with a group of minimalist real robots: Implementing secondary swarming," in IEEE International Conference on Systems, Man and Cybernetics, vol. 2, 2002, pp. 509-514.

[12] I. D. Kelly and D. D. A. Keating, "Flocking by the fusion of sonar and active infrared sensors on physical autonomous mobile robots," in In Proc. of the The Third Int. Conf. on Mechatronics and Machine Vision in Practice, 1996, pp. 1-4. 